\title{
Characterization of a novel polymeric Zinc (II) complex containing the anti- malarial Quinine as ligand: A Theoretical Approach (Semi-empirical and DFT methods).
}

\author{
I.A. Adejoro', O. E. Oyeneyin ${ }^{1}$, O.O. Adeboye ${ }^{1}$ and J. A. Obaleye ${ }^{2}$ \\ ${ }^{1}$ Department of Chemistry University of Ibadan, Ibadan, Nigeria. \\ 2Department of Chemistry, University of Ilorin, Ilorin, Nigeria.
}

\begin{abstract}
A novel polymeric zinc (II) complex of quinine, [chlorosulphato (2-ethenyl)-4-azabicyclo [2.2.2]oct5-ylium-(6-methoxyquinolin-4-yl) methanol zinc (II)] has been synthesized and characterized. However, theoretical studies on the geometries, thermodynamic parameters, vibrational frequencies, geometric parameters, dipole moments, and band gaps of the complex were carried out. All these properties were obtained using the AM1, PM3 and DFT methods. Comparisons were made on the calculated bond distances, bond angles, dihedral and vibrational frequencies with the experimental data and it compared favourably well.
\end{abstract}

Keywords: Geometric parameters, Zinc (II) Complex, Dipole moments, Band gaps, modelling.

\section{INTRODUCTION}

Computational chemistry is a useful way to investigate materials that are too difficult to find or too expensive to purchase. It also helps chemists make predictions before running the actual experiments so that they can be better prepared for making observations [1]. Molecular modeling is an aspect of computational chemistry. A particular molecular system can be modeled with the hope that it could be synthesized in the laboratory, especially for species that are too difficult, dangerous, impossible or too expensive to carry out experimentally. Molecular modeling is a quite accurate method in accounting for properties like geometric and electronic structures, frequencies, chemical shifts, bond distances and bond angles [2]. Semi - empirical calculations were carried out on orotic acid mixed with zinc (II) dihydrate acetate in a neutral medium and it was observed that the calculated result agree well with the experimental result [3]. Also, Semi- empirical calculation were carried out on novel aminopyridino 1-4-П-cyclohexa-1,3-diene iron tricarbonyl complexes, it was observed from the calculations that the complex is thermodynamically stable[4].

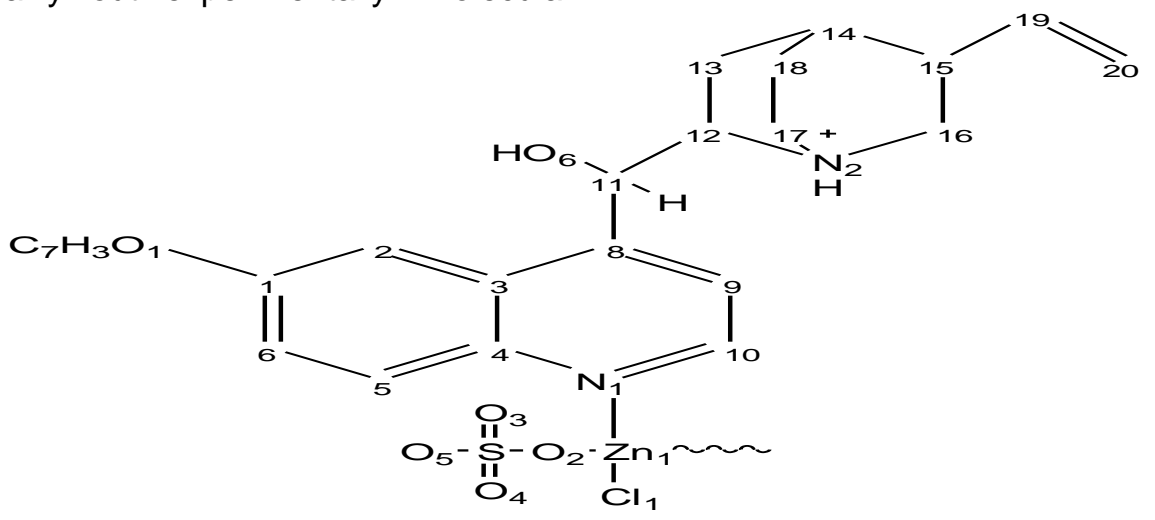

Fig. 1: The structure of the novel complex

This paper reveals calculated results relative to the experimental results that were carried out on the molecule [5] (figure1). The complex was modeled using Spartan'10 and theoretical calculations were carried out on the optimized geometries using Semi- empirical AM1, PM3 and DFT methods. The results were compared with each other and conclusion was made as to which of the methods compares favourably well with experimental data. 
Computational Methodology: The complex was modeled using Spartan'10. Semi-empirical and DFT molecular calculations were carried out on the complex. The structure was fully optimized.
Thermodynamic properties, vibrational frequencies, dipole moments, E HOMO, E LUMO, band gaps, heats of formation, and polarizabilities were carried out using these methods

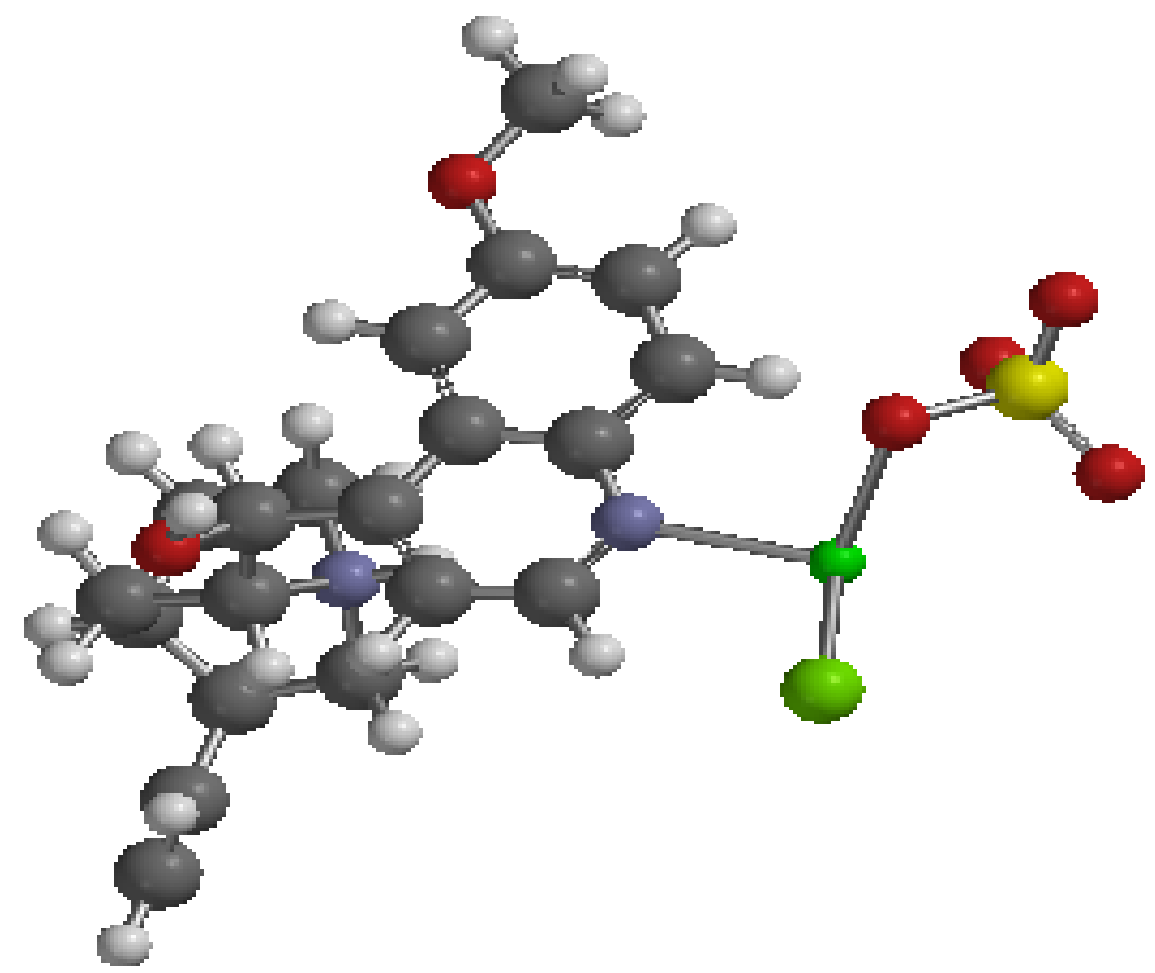

Fig. 2: The structure of the complex before optimization

\section{RESULTS AND DISCUSSION}

Geometric parameters: Calculations were carried out on the structure with the lowest energy values; this was obtained using conformer distribution calculation with $\mathrm{MMFF}_{\mathrm{aq}}$ [6]. Geometric parameters were obtained after optimization using $A M 1, P M 3$ and DFT $\left(6-31 G^{*}\right.$ and $\left.6-31 G^{\star *}\right)$. The bond distances, bond angles and dihedrals were measured compared with the experimental results (table 1). It was observed that PM3 result is closer to experimental result than AM1 but both could not account for the stability of the complex. The stability was accounted for using DFT with $6-31 G^{*}$ and $6-31 G^{* *}$. For instance the bond length between $\mathrm{Zn}_{1}-\mathrm{Cl}_{2}$ in the experimental result is 2.505 while in theoretical calculation, it is 2.093 (AMI), 2.285 (PM3), and 2.178 in DFT with 6-31G* and $6-31 G^{* *}$ respectively, between $Z n_{1}-O_{2}$, experimental result is 1.982 and in theoretical, it is 1.941 (AMI), 1.876 (PM3) and 1.975, 1.976 in DFT with $6-31 \mathrm{G}^{*}$ and $6-31 \mathrm{G}^{* *}$ respectively. Also, between $\mathrm{Zn}_{1}-\mathrm{N}_{1}$, the experimental result is 2.053 while in theoretical calculation it is 2.238 (AM1), 1.927 (PM3) and $2.162,2.160$ in DFT with $6-31 G^{*}$ and $6-31 G^{* *}$ respectively. The corresponding bond angles and dihedrals around the metal were also calculated as shown in tables 1, 2 and 3 and it was observed that the theoretical data compares well with the experimental data.

Electronic properties: The electronic structure of a crystal is described by its band structure [7]. This is obtained from the HOMO-LUMO calculations. The HOMO-LUMO band gap for this complex was 
calculated using AM1, PM3 and DFT $\left(6-31 \mathrm{G}^{*}\right.$ and 6$\left.31 \mathrm{G}^{* *}\right)$. The trend was that AMI $>$ DFT6-31G** PM3 $>$ DFT6-31G*. This shows that AM1 with band gap +8.03 predict the electronic properties of the complex better compare with other methods. Dipole moments was also calculated and the trend was PM3 $>$ DFT6-31G**>6-31G* $>$ AM1 as reported in table 4.

Thermodynamic properties and stabilities: For complexes to be thermodynamically stable, it is expected that $\Delta \mathrm{G}$ and $\Delta \mathrm{H}$ are negative. The more negative $\Delta G$ and $\Delta H$ are and the more positive $\Delta S$ is, the more stable the complex becomes. This paper with negative $\Delta \mathrm{G}, \Delta \mathrm{H}$ and positive $\Delta \mathrm{S}$ values as obtained from DFT calculations with $6-31 G^{*}$ and 6$31 \mathrm{G}^{\star *}$ basis set better predicts the stability of the polymeric Zinc (II) complex as shown in the thermodynamic properties in table 5. [8]

Vibrational Frequencies: The vibrational frequencies obtained from theoretical calculations were also in agreement with the experimental values as shown in table 6 that is absorption band $\mathrm{v}(\mathrm{O}-\mathrm{H})$ at $3745 \mathrm{~cm}^{-1}, \mathrm{v}(\mathrm{N}-\mathrm{H}) 3428 \mathrm{~cm}^{-1}, \mathrm{v}(\mathrm{C}-\mathrm{H}) 3247-3036 \mathrm{~cm}^{-1}$, $\mathrm{v}(\mathrm{C}=\mathrm{N}) 1515 \mathrm{~cm}^{-1}, \mathrm{v}(\mathrm{S}-\mathrm{O}) 1248-1244 \mathrm{~cm}^{-1}$ and the absorption band at $610 \mathrm{~cm}^{-1}$ for the involvement of quinuclidinic nitrogen. The theoretical values obtained using DFT with basis set $6-31 \mathrm{G}^{* *}$ are closer to experimental values.

\section{Geometric parameters:}

Bond distances (Å), Bond angles $\left(0^{\circ}\right)$ and Dihedrals $\left(0^{\circ}\right)$ of Zinc (II) Complex of Quinine, [Chlorosulphato (2-Ethenyl)4-Azabicyclo [2.2.2]Oct-5-ylium-(6 Methoxyquinolin-4-yl) Methanol Zinc (II)].

Table 1: Selected bond distances $(\AA)$ :

\begin{tabular}{|c|c|c|c|c|c|}
\hline Bond distance & Experimental & AM1 & PM3 & DFT/B3LYP (6-31G $\left.{ }^{*}\right)$ & DFT/B3LYP (6-31G**) \\
\hline $\mathrm{Zn}_{1}-\mathrm{Cl}_{1}$ & 2.505 & 2.093 & 2.285 & 2.178 & 2.178 \\
\hline $\mathrm{Zn}_{1}-\mathrm{O}_{2}$ & 1.982 & 1.941 & 1.876 & 1.975 & 1.976 \\
\hline $\mathrm{Zn}_{1}-\mathrm{N}_{1}$ & 2.053 & 2.238 & 1.927 & 2.162 & 2.160 \\
\hline $\mathrm{S}_{1}-\mathrm{O}_{2}$ & $\mathrm{~N} \backslash \mathrm{A}$ & 1.563 & 1.689 & 1.570 & 1.570 \\
\hline $\mathrm{S}_{1}-\mathrm{O}_{3}$ & $\mathrm{~N} \backslash \mathrm{A}$ & 1.455 & 1.440 & 1.469 & 1.468 \\
\hline $\mathrm{S}_{1}-\mathrm{O}_{4}$ & $\mathrm{~N} \backslash \mathrm{A}$ & 1.385 & 1.449 & 1.495 & 1.495 \\
\hline $\mathrm{S}_{1}-\mathrm{O}_{5}$ & $\mathrm{~N} \backslash \mathrm{A}$ & 1.423 & 1.697 & 1.574 & 1.574 \\
\hline $\mathrm{N}_{1}-\mathrm{C}_{10}$ & $\mathrm{~N} \backslash \mathrm{A}$ & 1.324 & 1.334 & 1.322 & 1.322 \\
\hline $\mathrm{N}_{1}-\mathrm{C}_{4}$ & $\mathrm{~N} \backslash \mathrm{A}$ & 1.393 & 1.403 & 1.366 & 1.366 \\
\hline $\mathrm{C}_{9}-\mathrm{C}_{10}$ & $\mathrm{~N} \backslash \mathrm{A}$ & 1.436 & 1.419 & 1.407 & 1.407 \\
\hline $\mathrm{C}_{8}-\mathrm{C}_{9}$ & $\mathrm{~N} \backslash \mathrm{A}$ & 1.373 & 1.376 & 1.382 & 1.434 \\
\hline $\mathrm{C}_{3}-\mathrm{C}_{4}$ & $\mathrm{~N} \backslash \mathrm{A}$ & 1.432 & 1.420 & 1.434 & 1.414 \\
\hline $\mathrm{C}_{4}-\mathrm{C}_{5}$ & $\mathrm{~N} \backslash \mathrm{A}$ & 1.430 & 1.423 & 1.414 & 1.373 \\
\hline $\mathrm{C}_{5}-\mathrm{C}_{6}$ & $\mathrm{~N} \backslash \mathrm{A}$ & 1.373 & 1.371 & 1.373 & 1.419 \\
\hline $\mathrm{C}_{6}-\mathrm{C}_{1}$ & $\mathrm{~N} \backslash \mathrm{A}$ & 1.417 & 1.418 & 1.419 & 1.359 \\
\hline $\mathrm{C}_{1}-\mathrm{O}_{1}$ & $\mathrm{~N} \backslash \mathrm{A}$ & 1.380 & 1.374 & 1.359 & 1.424 \\
\hline $\mathrm{C}_{7}-\mathrm{O}_{1}$ & $\mathrm{~N} \backslash \mathrm{A}$ & 1.425 & 1.409 & 1.424 & 1.385 \\
\hline $\mathrm{C}_{1}-\mathrm{C}_{2}$ & $\mathrm{~N} \backslash \mathrm{A}$ & 1.389 & 1.392 & 1.385 & 1.429 \\
\hline $\mathrm{C}_{3}-\mathrm{C}_{8}$ & $\mathrm{~N} \backslash \mathrm{A}$ & 1.437 & 1.437 & 1.429 & 1.517 \\
\hline $\mathrm{C}_{8}-\mathrm{C}_{11}$ & $\mathrm{~N} \backslash \mathrm{A}$ & 1.502 & 1.511 & 1.517 & 1.435 \\
\hline $\mathrm{C}_{11}-\mathrm{O}_{6}$ & $\mathrm{~N} \backslash \mathrm{A}$ & 1.423 & 1.417 & 1.436 & 1.549 \\
\hline $\mathrm{C}_{11}-\mathrm{C}_{12}$ & $\mathrm{~N} \backslash \mathrm{N}$ & 1.558 & 1.568 & 1.550 & 1.532 \\
\hline $\mathrm{N}_{2}-\mathrm{C}_{12}$ & $\mathrm{~N} \backslash \mathrm{A}$ & 1.501 & 1.540 & 1.532 & 1.536 \\
\hline $\mathrm{C}_{12}-\mathrm{C}_{13}$ & $\mathrm{~N} \backslash \mathrm{A}$ & 1.543 & 1.539 & 1.536 & 1.513 \\
\hline $\mathrm{N}_{2}-\mathrm{C}_{16}$ & $\mathrm{~N} \backslash \mathrm{A}$ & 1.501 & 1.527 & 1.514 & 1.533 \\
\hline $\mathrm{N}_{2}-\mathrm{C}_{17}$ & $\mathrm{~N} \backslash \mathrm{A}$ & 1.497 & 1.510 & 1.533 & 1.538 \\
\hline $\mathrm{C}_{17}-\mathrm{C}_{18}$ & $\mathrm{~N} \backslash \mathrm{A}$ & 1.532 & 1.520 & 1.539 & 1.540 \\
\hline $\mathrm{C}_{14}-\mathrm{C}_{18}$ & $\mathrm{~N} \backslash \mathrm{A}$ & 1.528 & 1.535 & 1.540 & 1.555 \\
\hline $\mathrm{C}_{13}-\mathrm{C}_{14}$ & $\mathrm{~N} \backslash \mathrm{A}$ & 1.527 & 1.538 & 1.555 & 1.560 \\
\hline $\mathrm{C}_{14}-\mathrm{C}_{15}$ & $\mathrm{~N} \backslash \mathrm{A}$ & 1.537 & 1.548 & 1.560 & \\
\hline & & & & & \\
\hline
\end{tabular}


Table2: Selected bond angles: $\left({ }^{\circ}\right)$

\begin{tabular}{|c|c|c|c|c|c|}
\hline Bond angle & Experimental & AM1 & PM3 & DFT/B3LYP6-31G* & $\begin{array}{c}\text { DFT/B3LYP } \\
6-31 G^{\star *}\end{array}$ \\
\hline $\mathrm{Cl}_{1}-\mathrm{Zn}_{1}-\mathrm{O}_{2}$ & 119.2 & 154.89 & 125.07 & 139.02 & 139.03 \\
\hline $\mathrm{Cl}_{1}-\mathrm{Zn}_{1}-\mathrm{N}_{1}$ & 111.4 & 114.44 & 98.64 & 108.99 & 108.97 \\
\hline $\mathrm{O}_{2}-\mathrm{Zn}_{1}-\mathrm{N}_{1}$ & 95.2 & 89.97 & 119.35 & 103.00 & 103.01 \\
\hline $\mathrm{O}_{2}-\mathrm{S}_{1}-\mathrm{O}_{3}$ & 109.3 & 103.08 & 113.02 & 112.49 & 112.51 \\
\hline $\mathrm{O}_{2}-\mathrm{S}_{1}-\mathrm{O}_{5}$ & 103.5 & 108.19 & 90.68 & 99.62 & 99.62 \\
\hline $\mathrm{O}_{2}-\mathrm{S}_{1}-\mathrm{O}_{4}$ & 109.7 & 108.59 & 110.74 & 109.80 & 109.84 \\
\hline $\mathrm{O}_{3}-\mathrm{S}_{1}-\mathrm{O}_{5}$ & 111.3 & 103.85 & 112.35 & 111.80 & 111.79 \\
\hline $\mathrm{O}_{3}-\mathrm{S}_{1}-\mathrm{O}_{4}$ & 112.2 & 115.31 & 118.13 & 113.93 & 113.93 \\
\hline $\mathrm{O}_{5}-\mathrm{S}_{1}-\mathrm{O}_{4}$ & 110.5 & 116.78 & 108.54 & 108.22 & 108.17 \\
\hline $\mathrm{Zn}_{1}-\mathrm{O}_{2}-\mathrm{S}_{1}$ & 128.4 & 155.52 & 95.15 & 94.38 & 94.40 \\
\hline $\mathrm{C}_{4}-\mathrm{N}_{1}-\mathrm{C}_{10}$ & $N \backslash A$ & 118.40 & 120.13 & 118.89 & 118.91 \\
\hline $\mathrm{C}_{3}-\mathrm{C}_{4}-\mathrm{C}_{5}$ & $\mathrm{~N} \backslash \mathrm{A}$ & 119.04 & 121.19 & 119.32 & 119.32 \\
\hline $\mathrm{C}_{5}-\mathrm{C}_{6}-\mathrm{C}_{1}$ & $\mathrm{~N} \backslash \mathrm{A}$ & 119.72 & 119.88 & 119.98 & 119.97 \\
\hline $\mathrm{C}_{1}-\mathrm{O}_{1}-\mathrm{C}_{7}$ & $\mathrm{~N} \backslash \mathrm{A}$ & 116.48 & 118.13 & 118.88 & 118.86 \\
\hline $\mathrm{O}_{6}-\mathrm{C}_{11}-\mathrm{C}_{12}$ & $\mathrm{~N} \backslash \mathrm{A}$ & 103.77 & 104.24 & 102.28 & 102.30 \\
\hline $\mathrm{O}_{6}-\mathrm{C}_{11}-\mathrm{C}_{8}$ & $N \backslash A$ & 111.91 & 112.40 & 112.09 & 112.08 \\
\hline $\mathrm{C}_{15}-\mathrm{C}_{19}-\mathrm{C}_{20}$ & $\mathrm{~N} \backslash \mathrm{A}$ & 124.00 & 128.98 & 129.63 & 129.58 \\
\hline $\mathrm{C}_{2}-\mathrm{C}_{1}-\mathrm{C}_{6}$ & $\mathrm{~N} \backslash \mathrm{A}$ & 120.95 & 121.37 & 120.23 & 120.26 \\
\hline $\mathrm{C}_{1}-\mathrm{C}_{6}-\mathrm{C}_{5}$ & $\mathrm{~N} \backslash \mathrm{A}$ & 119.72 & 119.88 & 119.98 & 119.97 \\
\hline $\mathrm{C}_{4}-\mathrm{C}_{5}-\mathrm{C}_{6}$ & $N \backslash A$ & 120.87 & 119.32 & 120.98 & 120.97 \\
\hline $\mathrm{C}_{2}-\mathrm{C}_{3}-\mathrm{C}_{4}$ & $N \backslash A$ & 119.06 & 118.37 & 118.63 & 118.65 \\
\hline $\mathrm{C}_{1}-\mathrm{C}_{2}-\mathrm{C}_{3}$ & $\mathrm{~N} \backslash \mathrm{A}$ & 120.35 & 119.77 & 120.85 & 120.81 \\
\hline $\mathrm{C}_{15}-\mathrm{C}_{16}-\mathrm{N}_{2}$ & $\mathrm{~N} \backslash \mathrm{A}$ & 111.62 & 109.20 & 108.41 & 108.38 \\
\hline $\mathrm{C}_{12}-\mathrm{N}_{2}-\mathrm{C}_{17}$ & $\mathrm{~N} \backslash \mathrm{A}$ & 110.19 & 111.93 & 113.11 & 113.07 \\
\hline $\mathrm{N}_{2}-\mathrm{C}_{17}-\mathrm{C}_{18}$ & $\mathrm{~N} \backslash \mathrm{A}$ & 111.03 & 109.58 & 107.77 & 107.75 \\
\hline $\mathrm{C}_{17}-\mathrm{C}_{18}-\mathrm{C}_{14}$ & $\mathrm{~N} \backslash \mathrm{A}$ & 109.52 & 107.91 & 107.77 & 107.74 \\
\hline $\mathrm{C}_{14}-\mathrm{C}_{15}-\mathrm{C}_{16}$ & $N \backslash A$ & 108.38 & 107.39 & 107.18 & 107.18 \\
\hline $\mathrm{C}_{17}-\mathrm{N}_{2}-\mathrm{C}_{16}$ & $\mathrm{~N} \backslash \mathrm{A}$ & 108.36 & 108.43 & 109.47 & 109.51 \\
\hline $\mathrm{C}_{8}-\mathrm{C}_{9}-\mathrm{C}_{10}$ & $\mathrm{~N} \backslash \mathrm{A}$ & 119.24 & 119.86 & 119.78 & 119.78 \\
\hline $\mathrm{C}_{10}-\mathrm{N}_{1}-\mathrm{Zn}_{1}$ & $N \backslash A$ & 115.26 & 118.96 & 105.68 & 105.47 \\
\hline
\end{tabular}

Table 3: Selected Dihedrals:

\begin{tabular}{|c|c|c|c|c|c|}
\hline Dihedral & Experimental & AM1 & PM3 & $\begin{array}{c}\text { DFT/B3LYP } \\
6-31 G^{*}\end{array}$ & $\begin{array}{c}\text { DFT/B3LP } \\
6-31 G^{\star *}\end{array}$ \\
\hline $\mathrm{Zn}_{1}-\mathrm{O}_{2}-\mathrm{S}_{1}-\mathrm{O}_{3}$ & 71.7 & -1.57 & -114.21 & -124.34 & -124.25 \\
\hline $\mathrm{Zn}_{1}-\mathrm{O}_{2}-\mathrm{S}_{1}-\mathrm{O}_{5}$ & -169.6 & 108.01 & 0.51 & -5.80 & -5.71 \\
\hline $\mathrm{Zn}_{1}-\mathrm{O}_{2}-\mathrm{S}_{1}-\mathrm{O}_{4}$ & -51.7 & -124.34 & 110.67 & 107.66 & 107.71 \\
\hline Cl1-Zn1-O2-S1 & $\mathrm{N} \backslash \mathrm{A}$ & 167.61 & 123.06 & 137.41 & 137.26 \\
\hline Cl1-Zn1-N1-C4 & $\mathrm{N} \backslash \mathrm{A}$ & 78.12 & -93.02 & 149.86 & 150.38 \\
\hline Cl1-Zn1-N1-C10 & $\mathrm{N} \backslash \mathrm{A}$ & -132.45 & 66.54 & -73.30 & -72.95 \\
\hline $\mathrm{C} 7-\mathrm{O} 1-\mathrm{C} 1-\mathrm{C} 2$ & $\mathrm{~N} \backslash \mathrm{A}$ & 179.32 & 177.23 & -177.07 & -177.03 \\
\hline O6-C11-C12-N2 & $\mathrm{N} \backslash \mathrm{A}$ & -157.85 & -104.13 & -53.03 & -53.05 \\
\hline C20-C19-C15-C14 & $\mathrm{N} \backslash \mathrm{A}$ & -168.57 & -125.30 & -128.93 & -129.23 \\
\hline C20-C19-C15-C16 & $\mathrm{N} \backslash \mathrm{A}$ & -46.10 & -1.82 & -3.98 & -4.25 \\
\hline
\end{tabular}

${ }^{*} \mathrm{~N} \backslash \mathrm{A}=$ Experimental data not available 
Am. J. Sci. Ind. Res., 2013, 4(1): 111-122

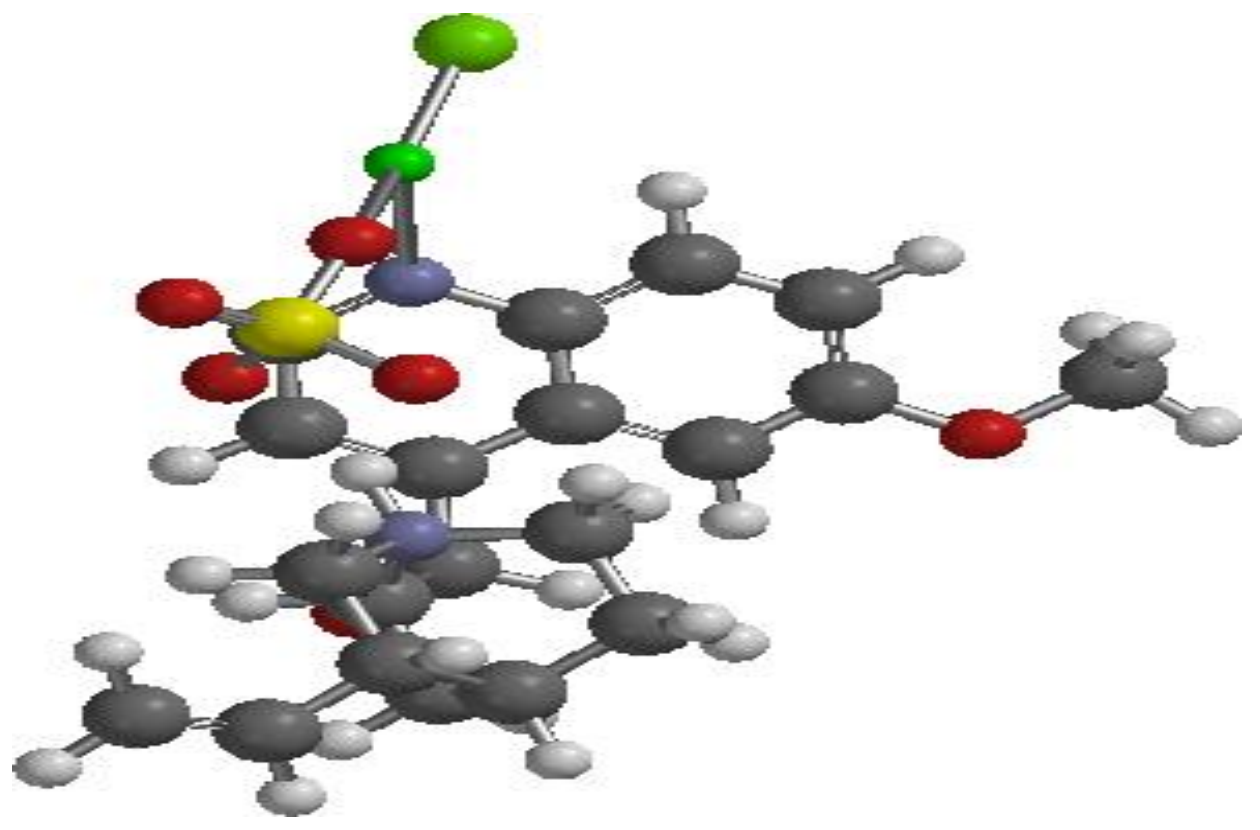

Fig. 3: The optimized structure of the complex using AM1

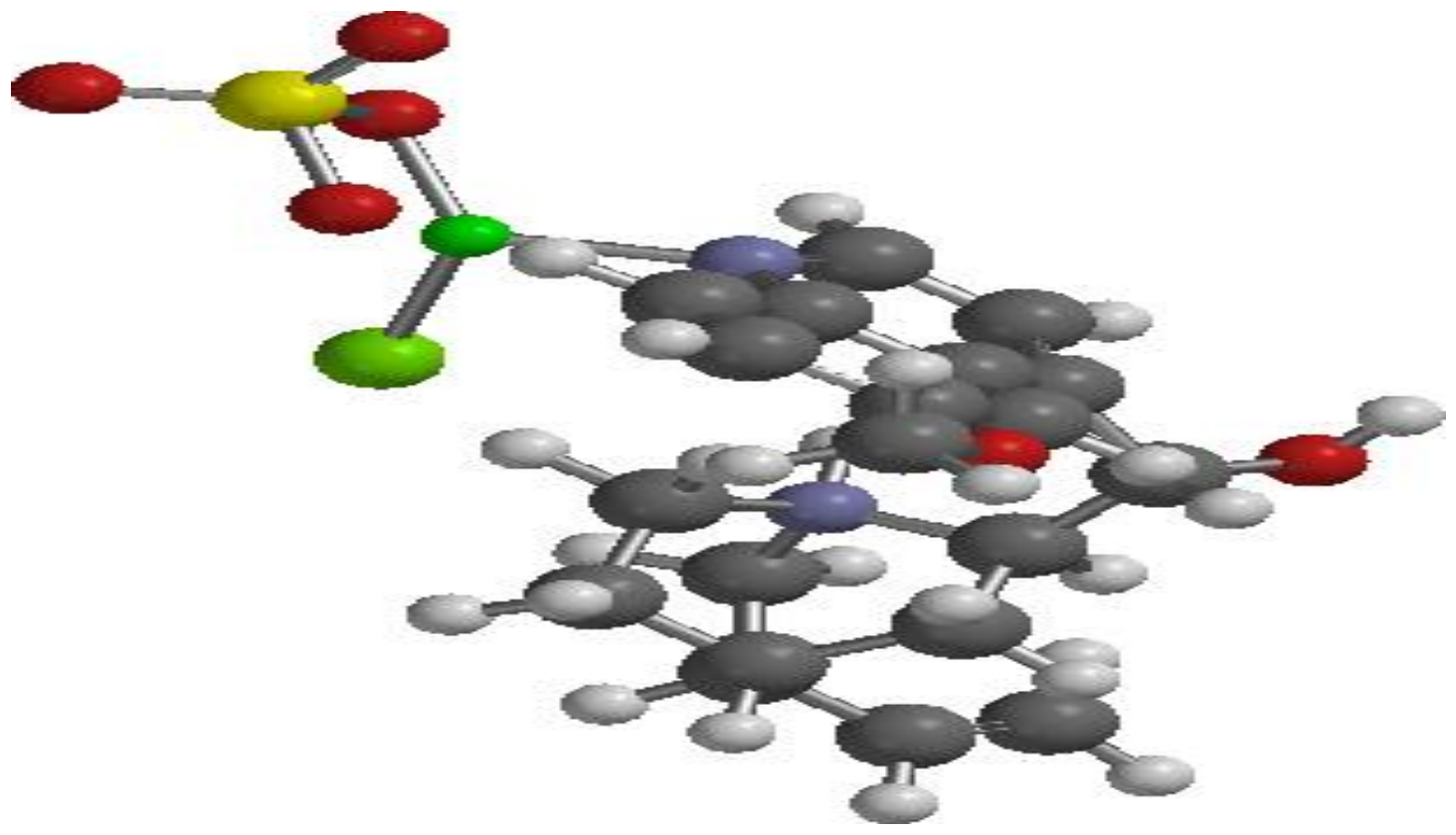

Fig. 4: The optimized structure of the complex with PM3 


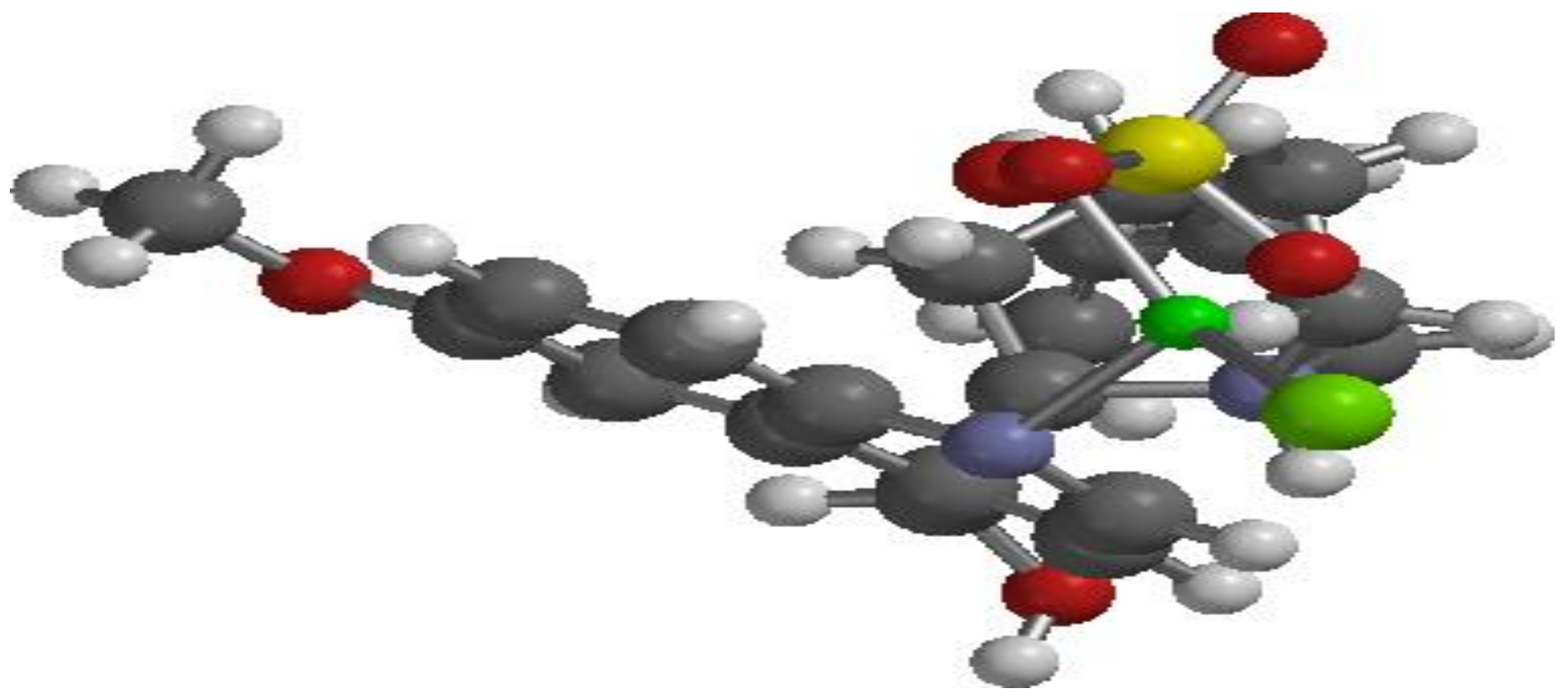

Fig. 5: The optimized structure of the complex using the DFT with B3LYP/6-31G*

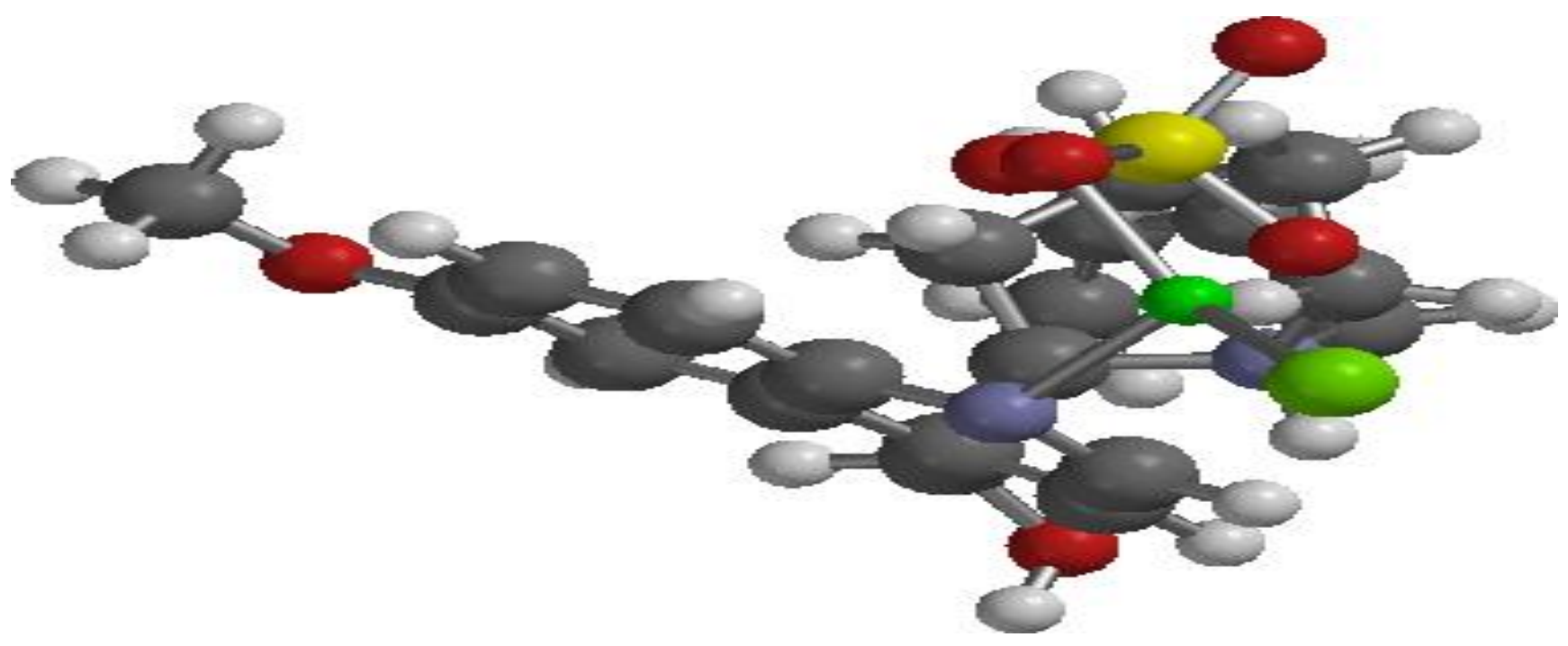

Fig. 6: The optimized geometry of the complex using the DFT with B3LYP/6-31G** 
Am. J. Sci. Ind. Res., 2013, 4(1): 111-122

Table 4: Dipole moments, HOMO and LUMO energies, Energy band gaps (Eg) for the complex

\begin{tabular}{|c|c|c|c|c|}
\hline Methods & $\begin{array}{c}\text { Dipole } \\
\text { moments/ } \\
\text { (Debye) }\end{array}$ & HOMO energy/eV & LUMO energy/Ev & Band gaps/eV \\
\hline AM1 & 15.39 & -9.52 & -1.49 & +8.03 \\
\hline PM3 & 25.99 & -9.34 & -2.22 & +7.12 \\
\hline $\begin{array}{c}\text { DFT } \\
\left(6-31 G^{\star}\right)\end{array}$ & 20.46 & -5.43 & -2.26 & +7.17 \\
\hline $\begin{array}{c}D F T \\
\left(6-31 G^{* *}\right)\end{array}$ & 20.74 & -8.59 & 0.76 & +7.83 \\
\hline
\end{tabular}

Table 5: Thermodynamics parameters for the complex at $298.15 \mathrm{~K}$

\begin{tabular}{|c|c|c|c|c|c|}
\hline Methods & $\begin{array}{l}\text { Heat of } \\
\text { Formation } \\
\left(\mathrm{KJmol}^{-1}\right)\end{array}$ & $\begin{array}{l}\text { SCF Total } \\
\text { energy/Hartrees }\end{array}$ & Free energy/au & Enthalpy/au & $\begin{array}{l}\text { Entropy } \\
\left.\mathbf{( J m o l}^{-1} \mathbf{K}^{-1}\right)\end{array}$ \\
\hline AM1 & -694.21 & & 0.13 & 0.21 & 661.65 \\
\hline PM3 & -1160.08 & - & -0.05 & 0.02 & 650.73 \\
\hline $\begin{array}{c}\text { DFT } \\
\left(6-31 G^{\star}\right)\end{array}$ & - & -3975.53 & -3975.13 & -3975.06 & 665.58 \\
\hline $\begin{array}{c}\text { DFT } \\
\left(6-31 G^{* \star}\right)\end{array}$ & - & -3975.57 & -3964.11 & -3964.04 & 665.86 \\
\hline
\end{tabular}

Table 6: Vibrational frequencies showing absorption bands and intensities of the complex. $\left(\mathrm{cm}^{-1}\right)$

\begin{tabular}{|c|c|c|c|c|}
\hline $\begin{array}{c}\text { Experimental } \\
\text { Vib. Freq. }\end{array}$ & AM1 & PM3 & $\begin{array}{c}\text { DFT/B3LYP } \\
6-31 G^{*}\end{array}$ & $\begin{array}{l}\text { DFT/B3LYP } \\
6-31 G^{* *}\end{array}$ \\
\hline 435.14 & $436 \quad(207.52)$ & $438(15.81)$ & $437 \quad(3.96)$ & $436 \quad(3.92)$ \\
\hline 469.97 & $(9.41)$ & $468 \quad(2.47)$ & $468 \quad(0.59)$ & $468 \quad(0.55)$ \\
\hline 558.32 & $(9.38)$ & 552 (6.03) & $558 \quad(12.97)$ & $558 \quad(13.00)$ \\
\hline 602.97 & $(60.56)$ & $\mathrm{N} / \mathrm{A}$ & $610 \quad(0.05)$ & $609 \quad(0.05)$ \\
\hline 618.73 & $\mathrm{~N} / \mathrm{A}$ & $610 \quad(5.08)$ & $616 \quad(5.01)$ & $(5.10)$ \\
\hline 648.17 & $(41.22)$ & $640 \quad(1.55)$ & $647 \quad(66.85)$ & $\begin{array}{ll}647 & (66.85) \\
\end{array}$ \\
\hline 669.41 & $(89.78)$ & 665 (129.99) & $\begin{array}{ll}660 & (5.92)\end{array}$ & $660 \quad(6.01)$ \\
\hline 718.44 & $715 \quad(110.45)$ & $716 \quad(3.12)$ & $\begin{array}{ll}707 & (11.77)\end{array}$ & $\begin{array}{ll}707 & (11.54)\end{array}$ \\
\hline 803.79 & $803 \quad(8.01)$ & $811 \quad(2.74)$ & $807 \quad(17.43)$ & $\begin{array}{ll}806 & (17.72) \\
\end{array}$ \\
\hline 835.67 & (3.09) & $(31.79)$ & $828 \quad(15.25)$ & $827 \quad(20.10)$ \\
\hline 861.01 & $(5.08)$ & $(21.32)$ & $860 \quad(92.28)$ & $859 \quad(103.18)$ \\
\hline 877.36 & $879 \quad(525.35)$ & $(6.61)$ & $875 \quad(9.70)$ & $875 \quad(9.43)$ \\
\hline 920.30 & $917 \quad(8.74)$ & $(103.41)$ & $922 \quad(8.66)$ & (8.51) \\
\hline 976.83 & $(3.80)$ & $(7.87)$ & $981 \quad(0.32)$ & $(8.46)$ \\
\hline 1014.91 & $1017 \quad(8.58)$ & $(9.37)$ & 1017 (13.27) & 1014 (12.76) \\
\hline 1115.42 & 1118 (13.47) & $(1.16)$ & $1125(32.12)$ & $1120 \quad(2.76)$ \\
\hline 1186.36 & $1192(7.48)$ & (4.27) & $1188(24.13)$ & 1185 (11.15) \\
\hline 1252.40 & $1267(4.11)$ & $(39.38)$ & $1248(311.78)$ & $1248(316.33)$ \\
\hline 1321.85 & 1334 (165.93) & $(87.87)$ & $1316(315.13)$ & 1327 (13.77) \\
\hline 1367.82 & 1367 (86.34) & $(46.07)$ & $1370(26.14)$ & 1375 (16.20) \\
\hline 1434.86 & $1434 \quad(50.58)$ & $(8.46)$ & $1436(15.20)$ & 1430 (13.93) \\
\hline 1474.76 & $1488(0.33)$ & $(29.84)$ & $1473(42.82)$ & $1475(15.00)$ \\
\hline 1517.67 & $\mathrm{~N} / \mathrm{A}$ & $(26.40)$ & $1515(9.12)$ & 1518 (16.22) \\
\hline 1594.38 & $\mathrm{~N} / \mathrm{A}$ & $(77.67)$ & $\mathrm{N} / \mathrm{A}$ & $\mathrm{N} / \mathrm{A}$ \\
\hline 1621.86 & $\mathrm{~N} / \mathrm{A}$ & $\mathrm{N} / \mathrm{A}$ & 1625 (8.69) & $1622(9.83)$ \\
\hline 2662.91 & $\mathrm{~N} / \mathrm{A}$ & $\mathrm{N} / \mathrm{A}$ & $\mathrm{N} / \mathrm{A}$ & $\mathrm{N} / \mathrm{A}$ \\
\hline 2975.34 & $\mathrm{~N} / \mathrm{A}$ & $2970 \quad(17.05)$ & $\mathrm{N} / \mathrm{A}$ & $\mathrm{N} / \mathrm{A}$ \\
\hline 3360.76 & $\mathrm{~N} / \mathrm{A}$ & $\mathrm{N} / \mathrm{A}$ & $\mathrm{N} / \mathrm{A}$ & $\mathrm{N} / \mathrm{A}$ \\
\hline
\end{tabular}


Table 7: Absorption Bands with their corresponding Vibration. $\left(\mathrm{cm}^{-1}\right)$

\begin{tabular}{|c|c|c|c|c|c|}
\hline Vibrations & Experimental & $\begin{array}{r}\text { DFT/B3LYP } \\
\text { 6-31G** }\end{array}$ & $\begin{array}{r}\text { DFT/B3LYP } \\
6-31 G^{* *}\end{array}$ & PM3 & AM1 \\
\hline $\mathrm{O}-\mathrm{H}$ bending & 1620 & - & - & - & - \\
\hline $\mathrm{O}-\mathrm{H}$ Stretching & $3364-3211$ & 3745 & 3816 & 3893 & 2748 \\
\hline N-H Stretching & 2575and 2662 & 3428 & 3440 & 3267 & $\mathrm{~N} / \mathrm{A}$ \\
\hline C-H Stretching & 2975 and 2900 & $3247-3036$ & 3816 & 3893 & 2748 \\
\hline \multirow[t]{2}{*}{$\mathrm{C}=\mathrm{N}$ Stretching } & 1509 and 1518 & 1515 & 1509 & 1528 & 1446 \\
\hline & $\mathrm{N} / \mathrm{A}$ & $\mathrm{N} / \mathrm{A}$ & $\mathrm{N} / \mathrm{A}$ & $1870-1680$ & $\mathrm{~N} / \mathrm{A}$ \\
\hline S-O Stretching & $\begin{array}{c}977,1015,1115 \\
\text { and } 1186\end{array}$ & $1248-1244$ & 1248 & $\mathrm{~N} / \mathrm{A}$ & $668-627$ \\
\hline $\begin{array}{l}\text { Involvement of the } \\
\text { quinuclidinic nitrogen }\end{array}$ & 603,619 and 644 & 610 & 69 & 610 & $\mathrm{~N} / \mathrm{A}$ \\
\hline
\end{tabular}

The Infra-Red spectra showing the vibrations with their corresponding absorption band for AM1, PM3, DFT with B3LYP 6$31 \mathrm{G}^{*}$ and $6-31 \mathrm{G}^{* *}$ is as shown in figures: $\mathrm{a}, \mathrm{b}, \mathrm{c}, \mathrm{d}$, the ${ }^{1} \mathrm{H}$ NMR (table 7 ) and ${ }^{13} \mathrm{C}$ NMR spectra is also shown in figure e, $\mathrm{f}$ and $g$.

IR Spectrum $(1 / \mathrm{cm})$

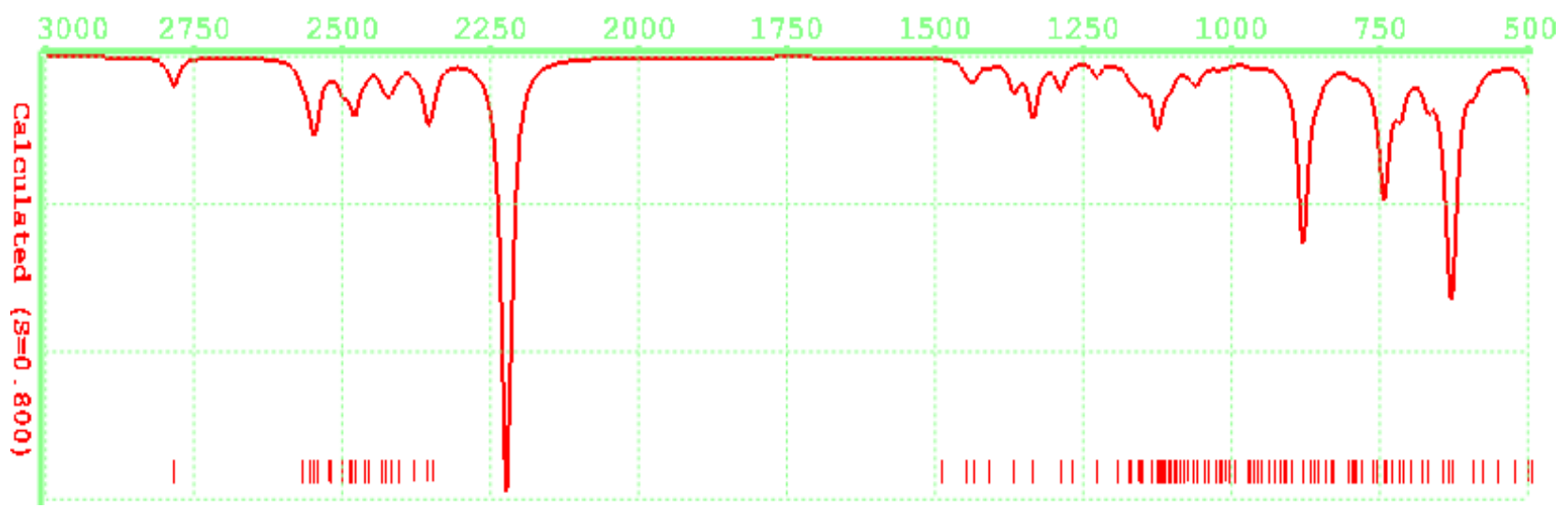

Fig a: The IR spectrum of the complex with AM1

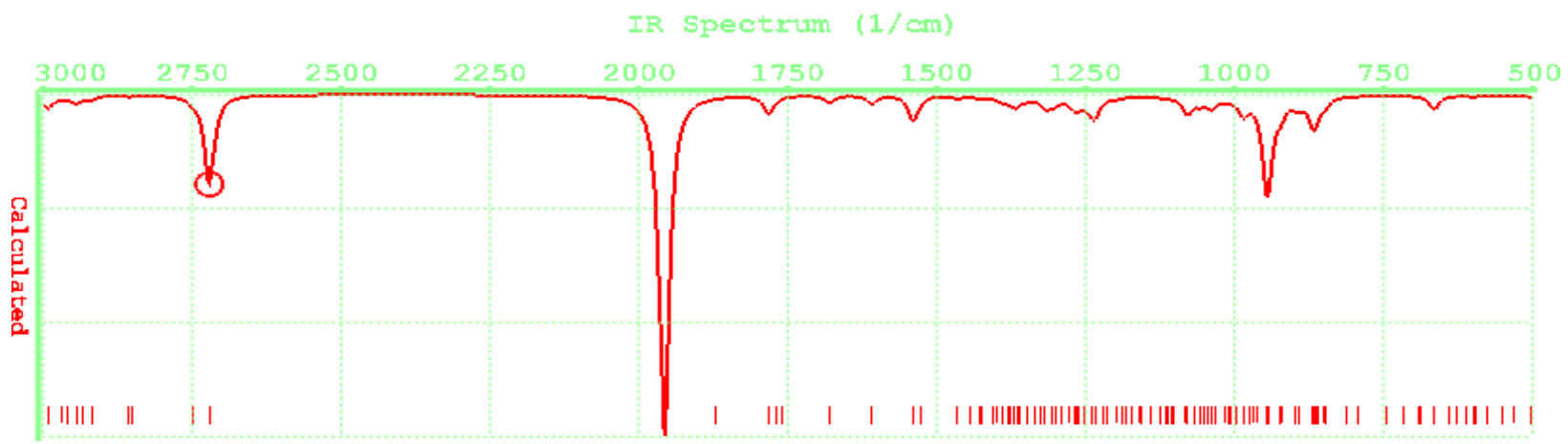

Fig b: The IR spectrum of the complex with PM3 
Am. J. Sci. Ind. Res., 2013, 4(1): 111-122

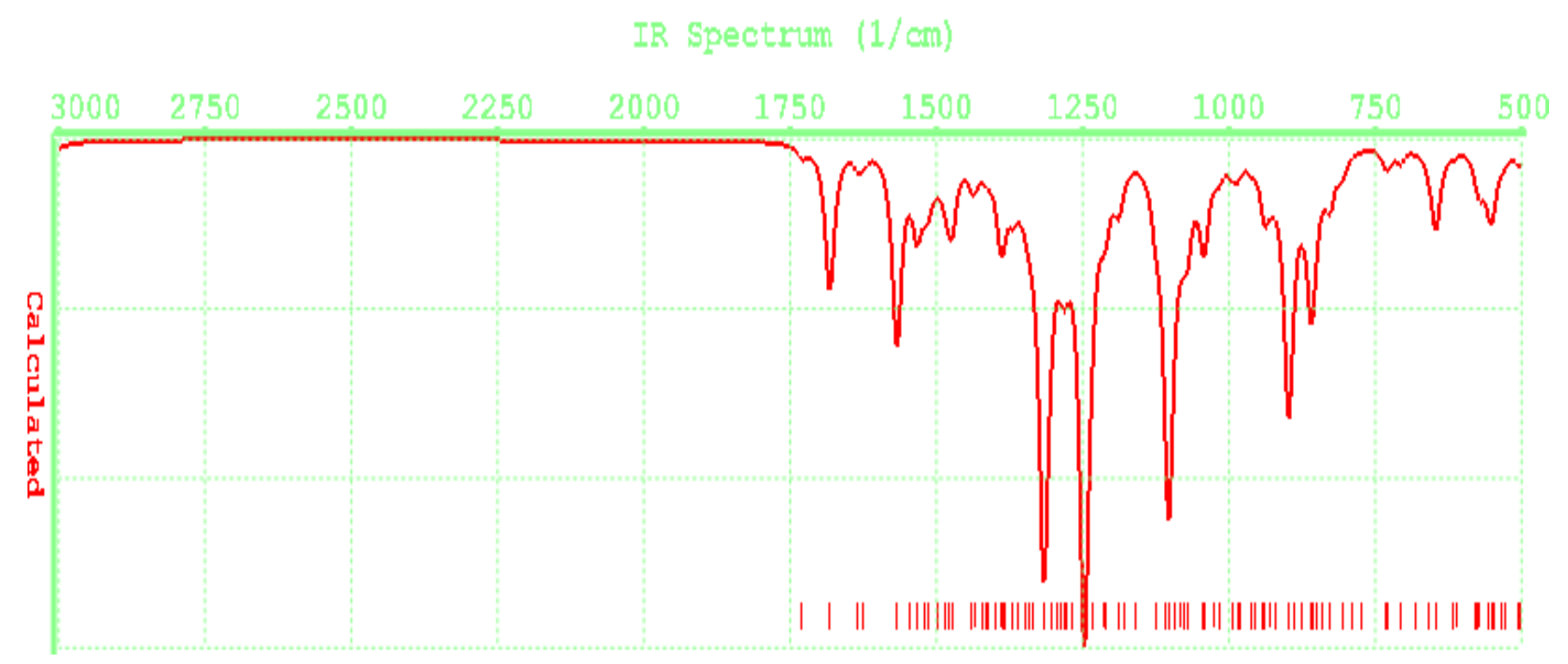

Fig. c The IR spectra of the complex with DFT B3LYP 6-31G*

\section{IR Spectrum $(1 / \mathrm{cm})$}

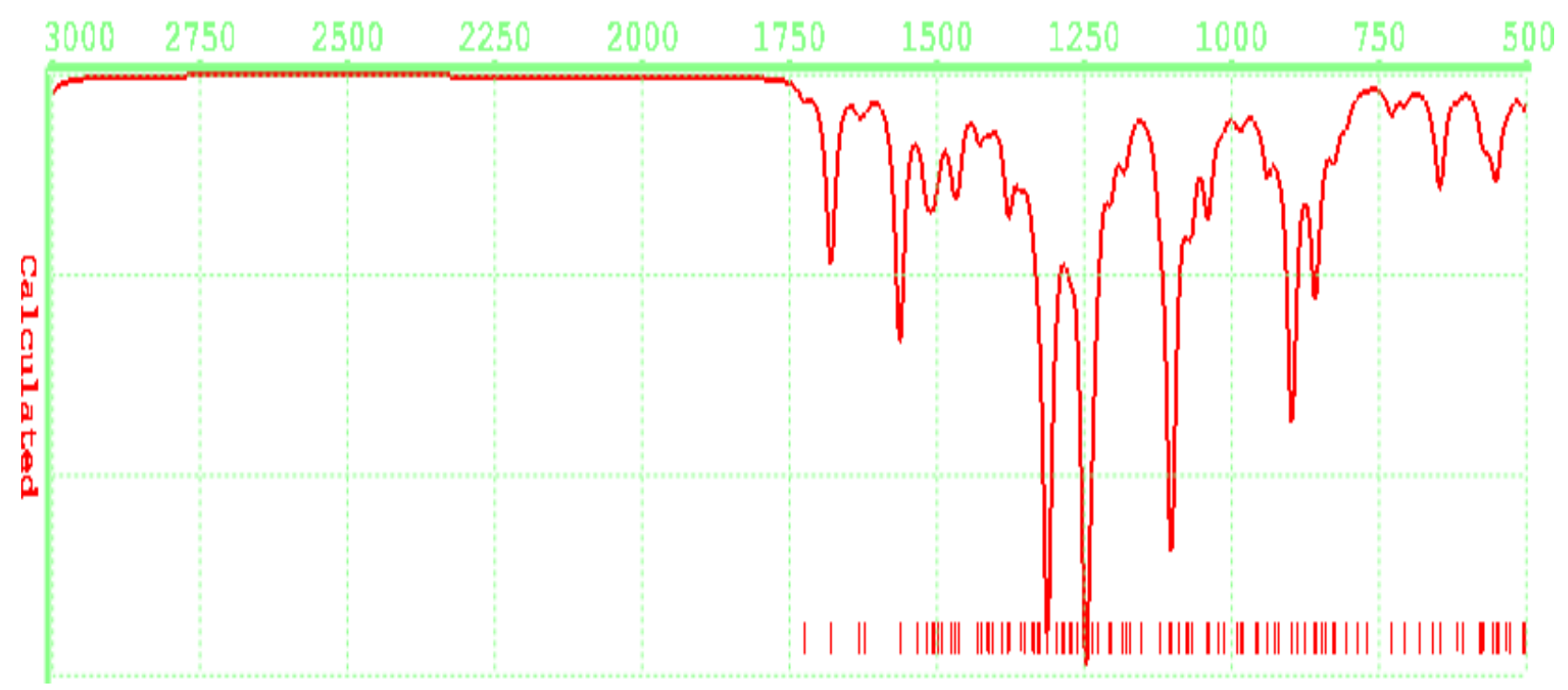

Fig. $d$ The IR spectrum of the complex with DFT B3LYP 6-31G** 
Table 8: NMR shifts (ppm) with DFT B3LYP 6-31G*

\begin{tabular}{|c|c|c|}
\hline Atom & Isotropic & Rel. Shift \\
\hline $1 \mathrm{C} 1$ & 38.362 & 151.26 \\
\hline $2 \mathrm{C} 2$ & 89.4777 & 100.15 \\
\hline $3 \mathrm{H} 6$ & 25.4982 & 6.69 \\
\hline $4 \mathrm{C3}$ & 66.7708 & 122.86 \\
\hline $5 \mathrm{C} 4$ & 52.1781 & 137.45 \\
\hline 6 C5 & 58.3248 & 131.30 \\
\hline $7 \mathrm{H} 10$ & 23.7522 & 8.43 \\
\hline $8 \mathrm{C} 6$ & 78.6768 & 110.95 \\
\hline $9 \mathrm{H} 14$ & 24.8956 & 7.29 \\
\hline 1001 & 231.1364 & $\mathrm{~N} \backslash \mathrm{A}$ \\
\hline $11 \mathrm{C7}$ & 136.6722 & 52.95 \\
\hline $12 \mathrm{H} 2$ & 28.4259 & 3.76 \\
\hline $13 \mathrm{H} 3$ & 28.1643 & 4.02 \\
\hline $14 \mathrm{H} 4$ & 28.2812 & 3.90 \\
\hline 15 C8 & 55.7062 & 133.92 \\
\hline $16 \mathrm{C9}$ & 80.9742 & 108.65 \\
\hline $17 \mathrm{H} 11$ & 25.1966 & 6.99 \\
\hline $18 \mathrm{C} 10$ & 46.6229 & 143.00 \\
\hline $19 \mathrm{H} 12$ & 23.3553 & 8.83 \\
\hline $20 \mathrm{~N} 1$ & -19.6478 & -98.14 \\
\hline $21 \mathrm{Zn} 1$ & 1899.6846 & $\mathrm{~N} \backslash \mathrm{A}$ \\
\hline $22 \mathrm{Cl} 1$ & 1096.6990 & $\mathrm{~N} \backslash \mathrm{A}$ \\
\hline $23 \mathrm{O} 2$ & 99.4152 & $\mathrm{~N} \backslash \mathrm{A}$ \\
\hline $24 \mathrm{~S} 1$ & 191.3937 & $\mathrm{~N} \backslash \mathrm{A}$ \\
\hline 2503 & 110.4351 & $\mathrm{~N} \backslash \mathrm{A}$ \\
\hline 2604 & 112.3272 & $N \backslash A$ \\
\hline 2705 & 77.4991 & $N \backslash A$ \\
\hline $28 \mathrm{C} 11$ & 121.4490 & 68.18 \\
\hline $29 \mathrm{H} 1$ & 26.5883 & 5.60 \\
\hline 3006 & 279.9944 & $\mathrm{~N} \backslash \mathrm{A}$ \\
\hline $31 \mathrm{H} 5$ & 31.0339 & 1.15 \\
\hline 32 C12 & 122.5523 & 67.07 \\
\hline $33 \mathrm{H} 9$ & 28.2621 & 3.92 \\
\hline $34 \mathrm{C} 13$ & 168.5633 & 21.06 \\
\hline $35 \mathrm{H} 8$ & 29.1763 & 3.01 \\
\hline $36 \mathrm{H} 13$ & 30.5988 & 1.59 \\
\hline $37 \mathrm{C} 14$ & 159.1264 & 30.50 \\
\hline $38 \mathrm{H} 17$ & 30.0926 & 2.09 \\
\hline 39 C15 & 148.9226 & 40.70 \\
\hline $40 \mathrm{H} 15$ & 29.5818 & 2.60 \\
\hline $41 \mathrm{C} 16$ & 138.9460 & 50.68 \\
\hline $42 \mathrm{H} 20$ & 29.2295 & 2.95 \\
\hline $43 \mathrm{H} 21$ & 28.6205 & 3.56 \\
\hline $44 \mathrm{~N} 2$ & 206.4989 & -324.28 \\
\hline $45 \mathrm{H} 22$ & 27.3021 & 4.88 \\
\hline $46 \mathrm{C} 17$ & 137.4213 & 52.21 \\
\hline $47 \mathrm{H} 7$ & 28.2386 & 3.95 \\
\hline $48 \mathrm{H} 23$ & 29.5900 & 2.59 \\
\hline $49 \mathrm{C} 18$ & 164.7188 & 24.91 \\
\hline $50 \mathrm{H} 24$ & 28.5375 & 3.65 \\
\hline $51 \mathrm{H} 25$ & 31.0305 & 1.15 \\
\hline 52 C19 & 57.7935 & 131.83 \\
\hline $53 \mathrm{C} 20$ & 82.5168 & 107.11 \\
\hline $54 \mathrm{H} 16$ & 25.9734 & 6.21 \\
\hline $55 \mathrm{H} 18$ & 27.0180 & 5.17 \\
\hline $56 \mathrm{H} 19$ & 26.7510 & 5.43 \\
\hline
\end{tabular}

${ }^{\star} \mathrm{N} \backslash \mathrm{A}=$ Relative Shift not available 
Am. J. Sci. Ind. Res., 2013, 4(1): 111-122

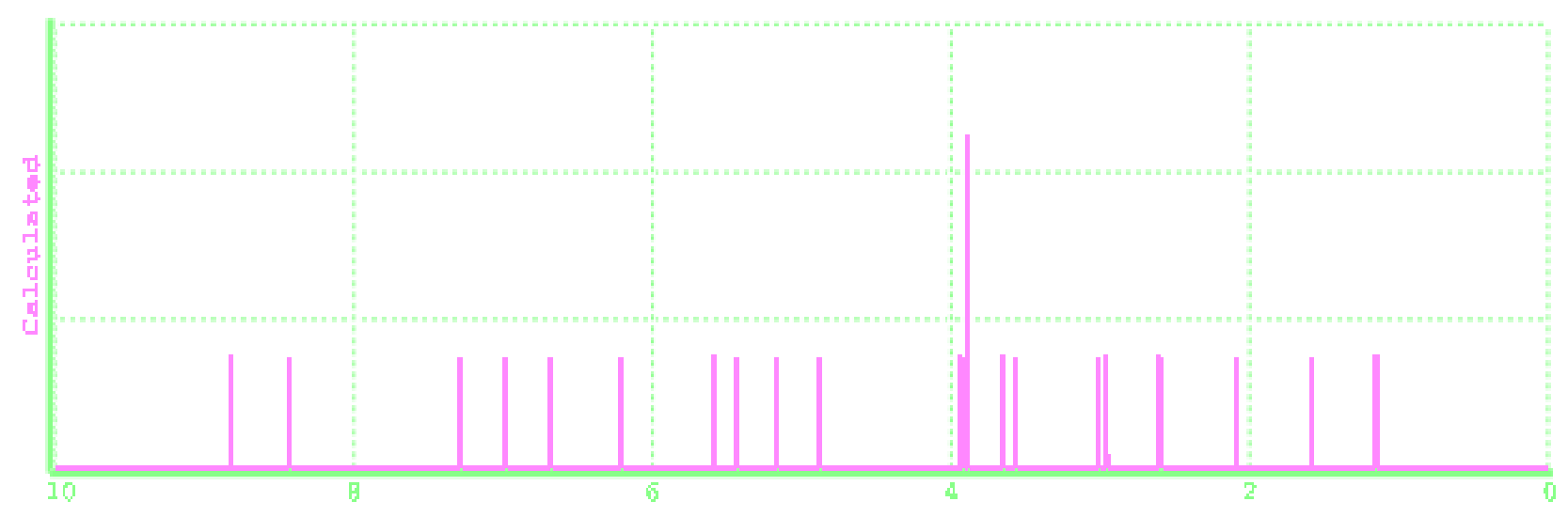

Fig e: ${ }^{1} \mathrm{H}-\mathrm{NMR}$ for the complex DFT B3LYP 6-31G*

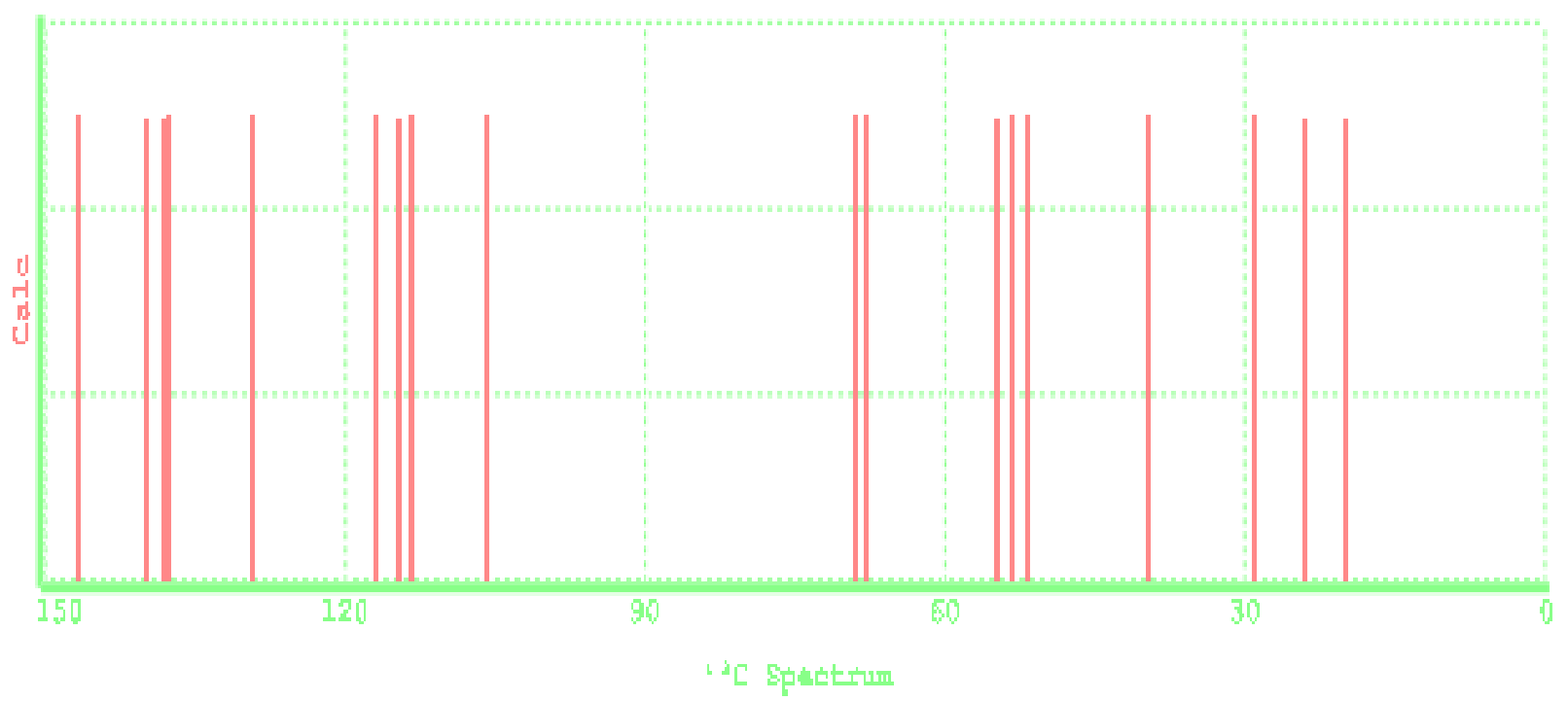

Fig f: ${ }^{13} \mathrm{C}$-NMR of the complex using DFT B3LYP 6-31G* and 6-31G**

\section{Conclusion}

The properties of the Complex [Chlorosulphato (2ethenyl)-4-Azabicyclo [2.2.2]Oct-5-ylium-(6Methoxyquinolin-4-yl) Methanol Zinc (II)] were calculated using Semi-empirical and DFT methods. The optimized geometries, dipole moments, geometric parameters, thermodynamic parameters and vibrational frequencies were investigated. Computational method has presented us the opportunity to take a critical look at this novel polymeric complex to produce results which compared favourably well with experimental data. It has also given us the opportunity to compute results on the properties that cannot be obtained in laboratory experiments.

Acknowledgement: $W e$ are grateful to the University of Ibadan for providing a good atmosphere for this research work. 


\section{REFERENCES}

1. Shodor (1999-2000) Overview of Computational Chemistry. http://www.shodor.org/chemviz/overview/ccbasica ssess.html

2. Conradie M.M (2010) Rhodium and Iron Complexes and Transition States: A Computational, Spectroscopic and Electrochemical Study. PhD thesis in the Department of Chemistry, Faculty of Natural and Agricultural Sciences. University of the Free State.

3. Gloria V.Seguel, Bernabe, L Rivas,Cesar Paredes (2010) Synthesis and Characterization of $\mathrm{Zn}$ (II) Complex with the Acetate and Orotic Acid Mix Ligands. J.Chil Chem Soc. 35(1). 5-7

4. Odiaka, T.I., Adejoro, I. A., and Akinyele, O. F. (2012) Semi- empirical (PM3) studies of novel aminopyridino - 1-4-П-cyclohexa-1, 3-diene iron tricarbonyl complexes. Am. J. Sci. Indi, Res. $3(1): 1-13$

5. Joshua A Obaleye, Mino R. Caira (2007) Synthesis, Characterization and Crystal Structure of a Polymeric $\mathrm{Zn}$ (II) Complex Containing the Antimalaria Qunine as ligand J. Chem Crystallogr 37: 707-712.

6. Warren J Hehre (2003) A guide to molecular mechanics and Quantum Chemical calculations in Spartan. Wavefunction, Inc. ISBN 1-890661$18-X 58$

7. David C Young (2001) Computational ChemistryA practical guide for applying real world problems. Wiley-interscience. ISBN 0-471-333689

8. Catherine E. Housecroft, Alan G. Sharpe (2005) Inorganic Chemistry, 2nd edition. Pearson Educational Limited, England. ISBN 0130-399132

9. Warren Hehre and Sean Ohlinger (2010) Spartan '10 Tutorial and user's guide. Wavefunction Inc. ISBN978-1-890661-41-4 